\title{
Fragmentation, Merging, and Internal Dynamics for PIC Simulation with Finite Size Particles
}

Dennis W. Hewett and David J. Larson

This article was presented at The 18th International Conference on Numerical Simulation of Plasmas (ICNSP '03) held on September 7-10, 2003 at the Sea Crest Oceanfront Resort and Conference Center in Cape Cod, Massachusetts

\section{August 11, 2003}

Lawrence

Livermore

National

Laboratory 


\section{DISCLAIMER}

This document was prepared as an account of work sponsored by an agency of the United States Government. Neither the United States Government nor the University of California nor any of their employees, makes any warranty, express or implied, or assumes any legal liability or responsibility for the accuracy, completeness, or usefulness of any information, apparatus, product, or process disclosed, or represents that its use would not infringe privately owned rights. Reference herein to any specific commercial product, process, or service by trade name, trademark, manufacturer, or otherwise, does not necessarily constitute or imply its endorsement, recommendation, or favoring by the United States Government or the University of California. The views and opinions of authors expressed herein do not necessarily state or reflect those of the United States Government or the University of California, and shall not be used for advertising or product endorsement purposes.

This is a preprint of a paper intended for publication in a journal or proceedings. Since changes may be made before publication, this preprint is made available with the understanding that it will not be cited or reproduced without the permission of the author. 


\title{
Fragmentation, Merging, and Internal Dynamics for PIC Simulation with Finite Size Particles
}

\author{
Dennis W. Hewett and David J. Larson \\ AX-Division \\ Lawrence Livermore National Laboratory
}

In plasma or rarified gas physics, collisions are rare but non-ignorable events. To model systems with arbitrary collisonality, it is necessary to start with a model that is fully capable of capturing collisionless, kinetic behavior. It is also necessary to build strategies to provide the essential economies into the scheme as collisions become more frequent. The desired model should progress smoothly and continuously from collisionless particle dynamics to collision-dominated fluid.

We are developing a new approach [Hewett, JCP 2003; Larson, JCP 2003] to recover the physics of this partially collisional regime. Our approach, called CPK (for Collisional Particle Kinetics) is basically a "smart particle" PIC scheme with particles that have internal parameters representing internal "fluid" behavior as the CPK particles become large via merging. In the collisionless limit, the individual macro particles become numerous, small and cold through fragmentation-leading naturally to the traditional PIC limit.

The new "smart" particle is a Gaussian distribution in all phase space directions. An arbitrary distribution of real particles can be made as a superposition of these "particles". One of the key capabilities is the ability to fragment each particle in a way that will not introduce new physics. With this procedure we can replace each particle with a set of particles that, when reassembled, give the original particle to arbitrary precision. Further, with some time-dependent parameters built into each particle, fragmentation is not required to preserve existing details during time evolution. Collisionless, free expansion of an isolated puff of gas can be followed by a single macro-particle with no fragmentation, if necessary. The redundancy introduced by the fragmentation provides the freedom to represent new features emerging from the nonlinear time evolution. In addition to collisionless internal particle dynamics, we have also developed internal dynamics consistent with a -law gas within each particle. This ability, coupled with a very aggressive strategy for merging particles with disparate velocities, puts full fluid behavior within reach.

The force between CPK particles is, presently, only through (perhaps aggressive) merging of $\mathrm{CPK}$ particles. A merger of two $\mathrm{CPK}$ particles is equivalent to an inelastic collision. The gradient of pressure is not computed and the "mesh" enters only in a nonfundamental way as a means to facilitate evaluating particle "overlap" in phase space in preparation for merging. The mesh never carries any essential part of the physics; the mesh could be discarded every time step if, for some reason, a new one offered advantages. 


\section{The CPK Macro-Particle}

Each CPK macro-particle is a grouping of real particles that have a Gaussian spatial profile about the central macro-particle Lagrangian location and a Maxwellian velocity profile about the Lagrangian drift. Each CPK particle has the form

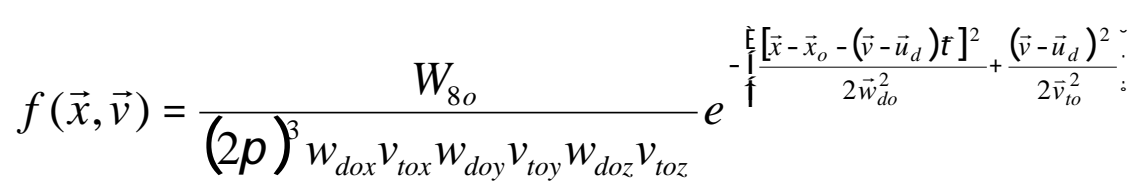

in which $W_{8 o}$ is the number of real particles, $\vec{w}_{d o}$ is the half-width in each space direction and $\vec{v}_{t o}$ is the thermal velocity (velocity half-width), and $\overrightarrow{\boldsymbol{x}}_{o}$ and $\vec{u}_{d}$ are the location and drift of the basic CPK particle. - represents the "age" of the distribution in the sense that, as the macro particle ages, free streaming causes shearing in phase space proportional to the net drift of that slice of the Gaussian with respect to the average $\vec{u}_{d}$. This is the simplest example of a parameter carried internally by the macro particle allowing physics to be retained by means other than simply adding more PIC particles. Other representations [Hewett, JCP 2003] enable internal capabilities such as internal evolution consistent with an arbitrary -law gas.

a) $=0$

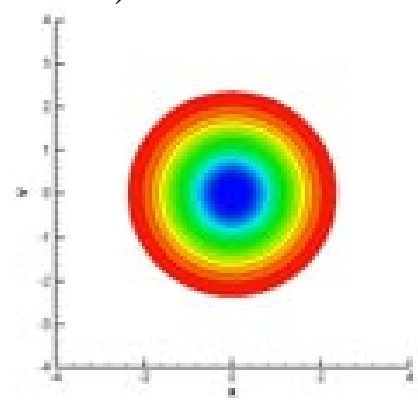

b) $=0.5$

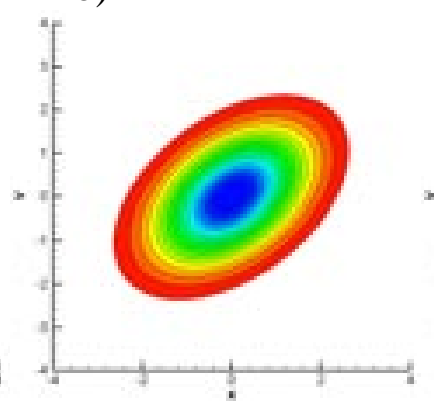

c) $=0.5$

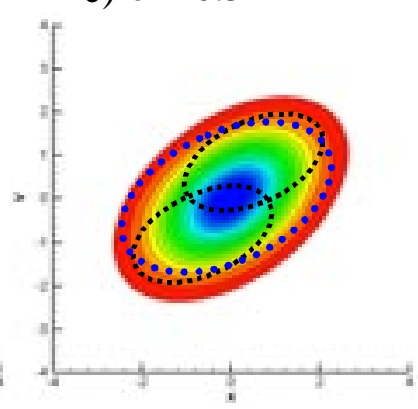

Shown in a) is the basic CPK particle form at $=0$; in b) is the same particle after a finite time ; and, in c) is the advanced particle with 3 velocity fragments outlined.

The simple spatial profile of the CPK macro particle makes techniques usually associated with the SPH (Smooth Particle Hydrodynamics) method a simple extension to the CPK method. However, SPH is a technique to solve the Eulerian fluid equations and, granted complete success, will only give Eulerian fluid solutions. CPK, on the other hand, is intended to recover the range of physics from the fully kinetic collisionless limit with multiple particles per cell to the fluid limit with just one Maxwellian particle at each grid point. 


\section{Adaptive Resolution Using CPK Macro-Particles}

CPK particles are used as a basis set from which the total distribution of real particles can be constructed by superposition. It is assumed that an arbitrary distribution of real particles can be represented by such a superposition, at least as well as could be done with the traditional PIC/MC delta functions and their associated "shape factors". We find that with this internal distribution, we can fragment each of the particles in either velocity or space such that the fragments could be reconstructed by superposition to recover the original. Thus we can increase the resolution of the macro particles locally, on demand, to recover emerging features of the total distribution. A key issue is that the act of fragmentation itself adds no additional features.

The increased resolution does cause additional expense and, as an economic necessity, we must be able to merge particles if interesting features fail to materialize. The Maxwellian velocity distribution within each particle is a feature that is useful for particle consolidation as collisionality relaxes the composite distribution towards a Maxwellian. Our merging scheme preserves the first 4 moments in both space and velocity.

\section{Applications}

As a first example, consider the following free expansion of a Gaussian puff of collisionless particles. (Hydrodynamics codes have difficulty "mass matching" zones for expansion into vacuum; Particle codes have not such difficulties.)
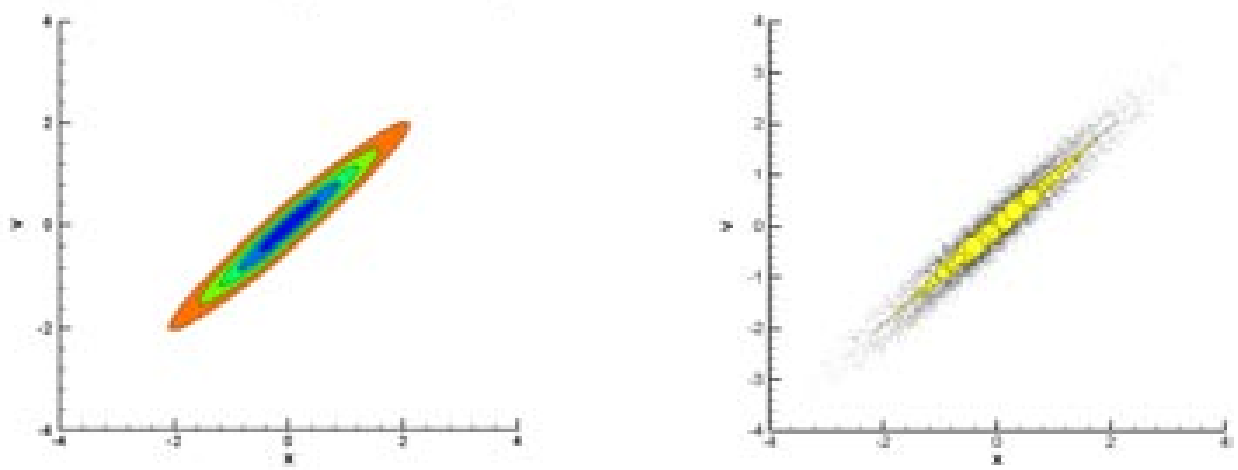

The evolution of a particle distribution representing a gas puff as it evolves from a vertical $=0$ state to a later time (left). On the right is a roughly 7000-particle distribution that started from one particle. Multiple fragmentations and merging have occurred, showing the desired larger particles in the center. Smaller particles, on the fringes, are "probing for emerging features".

Next, consider the shock tube problem (Sod, 1986) that consists of a collision-dominated gas held back by a membrane. Breaking the membrane at $\mathrm{t}=0$, the fluid moves towards the right and forms (from right to left) a shock front, a contact discontinuity, and a rarefaction wave. The pressure is constant across the contact discontinuity.

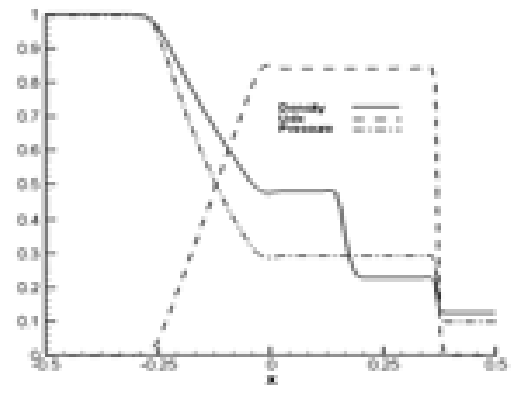


We model this problem with CPK by initializing the problem with a uniform density of CPK particles that have a greater weight and temperature on the left. The evolution begins by fragmentation of all particles that gives increased material flux to the right across the interface. Far from the interface, fragmentation is symmetric and merging preserves fluid homogeneity.
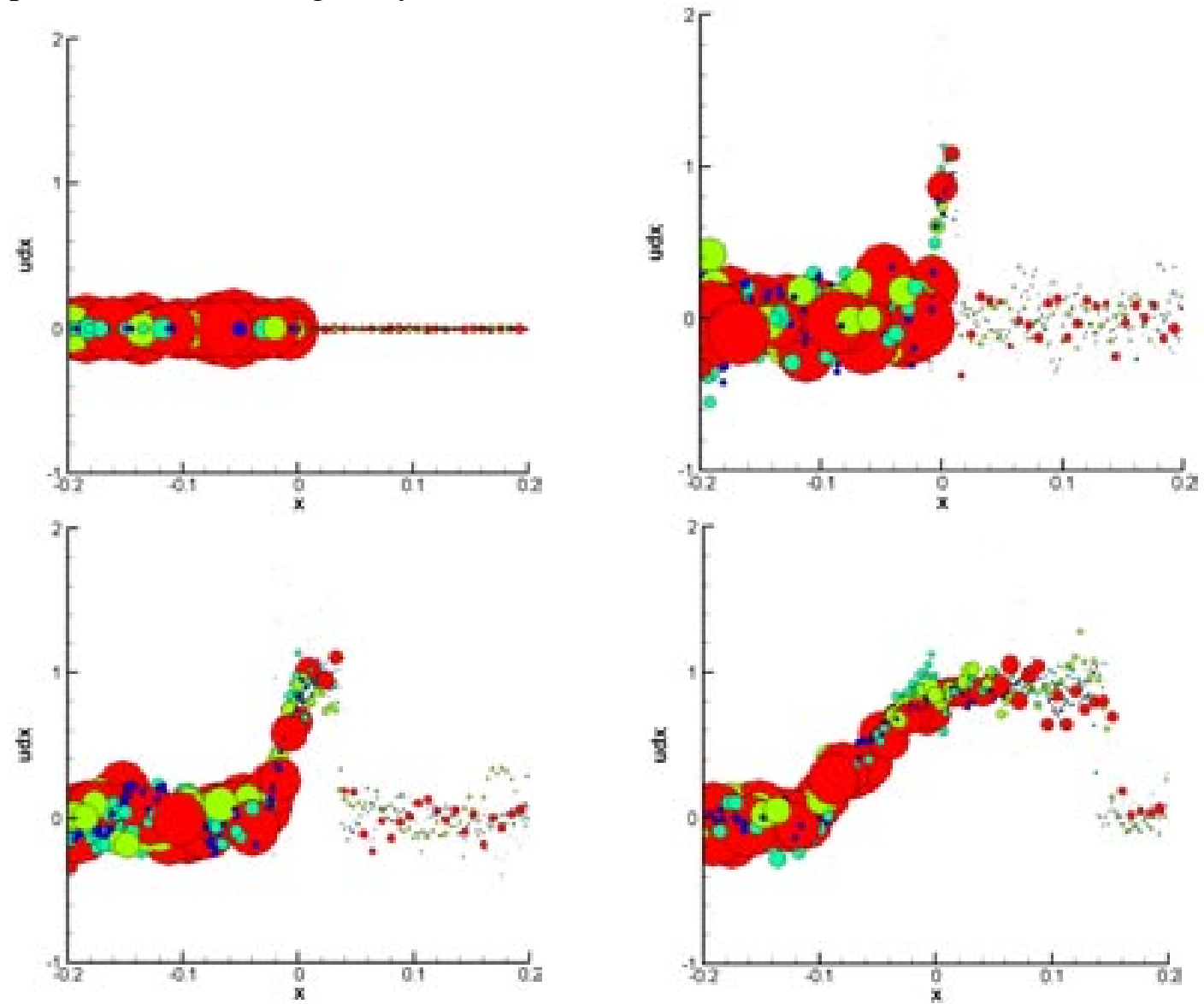

Show in the upper left is the particle phase space after the first time step. Probing for emerging features will soon detect (upper right), and encourage (lower left) a flow from a density gradient that results in particles developing a drift towards positive $x$. Later in the run the rarefaction has developed (lower right) and ultimately all the details of the Sod solution develop. Note particularly, the linear velocity ramp and the contact discontinuity showing higher temperature, lower density to the right of the contact discontinuity.

Also evident in the preceding figure is the color coding that denote various particle halfwidths. We are now investigating various schemes to do adaptive refinement that have to do with density gradients generated from particles of different sizes. Most important, we are actively pursuing a partially collisional technique (Larson and Hewett, these proceedings) that is compatible with the capabilities of CPK.

This work was performed under the auspices of the U.S. Department of Energy by the University of California, Lawrence Livermore National Laboratory under contract No. W-7405-Eng-48. 
University of California

Lawrence Livermore National Laboratory

Technical Information Department

Livermore, CA 94551

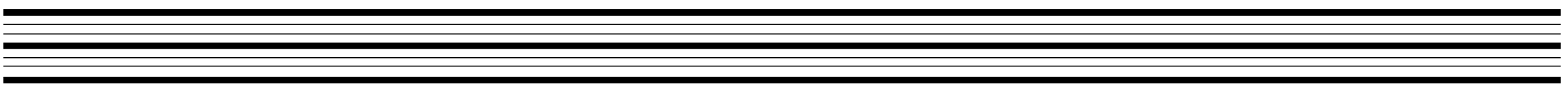

Th 